\title{
CHANNA STRIATUS TNFR-1: MOLECULAR CLONING, CHARACTERIZATION AND GENE EXPRESSION
}

\author{
Rajesh Palanisamy, Jesu Arockiaraj* \\ Division of Fisheries Biotechnology \& Molecular Biology, Department of Biotechnology, \\ Faculty of Science and Humanities, SRM University, Kattankulathur, Tamil Nadu, India.
}

\begin{abstract}
Tumor necrosis factor receptor (TNFR) superfamily entails in various biological functions including cell proliferation, differentiation, survival and providing co-stimulatory signals for programmed cell death or apoptosis. In this report, we have identified and characterized TNFR-1 from Channa striatus (Cs) at molecular level. In silico analyses revealed that the CsTNFR-1 cDNA sequence was 1200 bp in length, which includes an open reading frame of $1197 \mathrm{bp}$ that encoded a putative protein of 399 amino acids (45 $\mathrm{kDa}$ ). It contains three 'Cys' rich domains (CRDs) at CRD1 ${ }^{41-46}, \mathrm{CRD} 2^{79-118}$ and CRD3 ${ }^{120-159}$ in the extracellular region, also it possessed a putative TRAF6-binding site in the cytoplasmic tail. In addition, CsTNFR-1 cytoplasmic region contained a death domain between 297 and 388, which is important for apoptosis induction. It shared a maximum identity with its homologous from Paralichthys olivaceus (82\%). Real-time PCR findings revealed that CsTNFR-1 was expressed predominantly in head kidney, further it is up-regulated upon fungal (Aphanomyces invadans) and bacterial (Aeromonas hydrophila) infection. Overall, the gene expression results indicated that CsTNFR-1 involvement in the innate immune response against pathogenic infections. The recombinant CsTNFR-1 protein was over-expressed and purified using an Escherichia coli expression vector system. The purified recombinant CsTNFR-1 derivative binds with TNF- $\alpha$ at high affinity and inhibits its cytotoxic activity in vitro in a dose dependent manner. CsTNFR-1 was also analyzed for respiratory burst activity upon two different ROS inducers such as opsonized zymosan and phorbol 12-myristate 13-acetate (PMA). The results showed that the recombinant CsTNFR-1 protein alone did not produce any ROS, but upon induction with opsonized zymosan, it considerably increased the ROS production in a concentration dependent manner. Overall, the findings showed the potential involvement of CsTNFR-1 in the pathogen-induced inflammatory process of $C$. striatus.
\end{abstract}

\section{KEYWORDS}

Channa striatus; Tumor necrosis factor receptor 1; TNF- $\alpha$ inhibition activity; Cytotoxic activity; Respiratory burst activity.

* Corresponding author. Tel.: +91 44 27452270; fax: +91 4427453903.

E-mail address: jesuaraj@hotmail.com (J. Arockiaraj). 